\title{
EL GRUPO DE ACCIÓN FINANCIERA (GAFI) TREINTA AÑOS DESPUÉS: EL FUTURO DE LA LUCHA INTERNACIONAL CONTRA EL BLANQUEO DE CAPITALES Y LA FINANCIACIÓN DEL TERRORISMO THE FINANCIAL ACTION TASK FORCE (FATF) THIRTY YEARS AFTER: THE FUTURE OF THE INTERNATIONAL FIGHT AGAINST MONEY LAUNDERING AND THE FINANCING OF TERRORISM
}

\author{
Giorgios PAVLIDIS ${ }^{1}$
}

Recibido: $17 / 05 / 2020$

Aceptado: 13/06/2020

\begin{abstract}
SUMARIO: I. El grupo de acción financiera y la lucha internacional contra el blanqueo de capitales y la financiación del terrorismo; II. Difuminar la dicotomía entre el derecho vinculante y los instrumentos de carácter no vinculante; III. Garantizar la implementación universal de los estándares del GAFI; IV. Expansión de los estándares del GAFI; V. Sinergias entre el derecho nacional, el derecho internacional y las normas del GAFI; VI. ¿Creación de normas del GAFI o una nueva convención internacional? VII. Conclusiones; VIII. Bibliografía
\end{abstract}

SUMMARY: I. The financial action group and the international fight against money laundering and the financing of terrorism; II. The dicotomy between the non-binding and the binding law; III. The universal implementation of FATF standards; IV. The expansion of FATF standards; V. The synergies between national law, international law and FATF rules; VI. FATF standards or a new international convention? VII. Conclusions; VIII. Bibliography

Resumen: El Grupo de Acción Financiera (GAFI) constituye un excelente ejemplo del proceso de construcción de normas que trasciende las fuentes tradicionales y los mecanismos de aplicación del Derecho internacional público, al tiempo que exige un alto nivel de cumplimiento y asegura una rápida adaptación para abordar mejor el blanqueo de capitales y la financiación del terrorismo (AML/CFT por sus siglas en inglés). Con motivo del 30 aniversario del GAFI, vale la pena reexaminar el proceso de creación de sus normas, haciendo énfasis en su naturaleza legal, expansividad y flexibilidad, así como en los importantes desafíos que se avecinan debido a la naturaleza cambiante del blanqueo de capitales y de la financiación del terrorismo.

Palabras clave: Grupo de Acción Financiera, blanqueo de capitales, financiación del terrorismo.

Abstract: The Financial Action Task Force (FATF) constitutes a successful example of a normative process that goes beyond traditional sources and enforcement mechanisms of public international law and, at the same time, ensures high level of compliance and timely adaptation to challenges related to the fight against money laundering and

\footnotetext{
1 Profesor de Universidad Privada. Derecho Económico Internacional. Universidad NUP de Pafos (Chipre).
} 
terrorist financing (AML / CFT). On FATF's 30 ${ }^{\text {th }}$ anniversary, it is worth re-examining the characteristics of its norm-building model, in particular FATF's legal nature, its expansiveness and its flexibility. Such a review is important given the significant challenges posed by the evolving nature of money laundering and of terrorist financing.

Keywords: Financial Action Task Force, money laundering, financing of terrorism.

\section{EL GRUPO DE ACCIÓN FINANCIERA Y LA LUCHA INTERNACIONAL CONTRA EL BLANQUEO DE CAPITALES Y LA FINANCIACIÓN DEL TERRORISMO}

En el presente trabajo se ofrece un estudio acerca las contribuciones del Grupo de Acción Financiera (GAFI, en inglés: Financial Action Task Force, FATF) a la lucha internacional contra el blanqueo de capitales y el terrorismo (AML/CFT por sus siglas en inglés), se intenta comprender sus instrumentos como fuentes del Derecho y se expone la expansión inevitable de sus estándares ante les nuevas amenazas y desafíos en el contexto AML/CFT.

El GAFI es un organismo intergubernamental que se creó en 1989 en la $15^{\mathrm{a}}$ Cumbre Económica del Grupo de los Siete (G7) celebrada en París. El mandato inicial del GAFI, que luego se expandió gradualmente ${ }^{2}$, fue estudiar el fenómeno del blanqueo de capitales, sus tendencias y técnicas, a fin de proponer medidas para combatirlo. De acuerdo con tales objetivos, el GAFI habría completado su trabajo en 1990; sin embargo, los Estados miembros extendieron el mandato en varias ocasiones y, con motivo del 30 aniversario del GAFI en abril de 2019, acordaron un mandato abierto, reconociendo que el GAFI ha pasado de ser un foro temporal a un compromiso público y político sostenido para combatir el blanqueo de capitales, la financiación del terrorismo y la financiación de la proliferación de armas de destrucción masiva ${ }^{3}$.

La adopción de un mandato abierto para el GAFI no estuvo acompañada por la adopción de un tratado internacional que actuara como una Carta atribuyendo al GAFI un nuevo estatus legal como organización internacional pública. El modelo institucional existente se consideró un éxito que no requería de revisión. El nuevo mandato se limitó a introducir ajustes menores a la gobernanza del GAFI (p. ej. los ministros del GAFI se reunirían cada dos años y el mandato de la presidencia del GAFI también se extendió a un período de dos años). Además, se acordó un nuevo modelo de financiación para permitir que el GAFI se enfrente con eficacia a una carga de trabajo en expansión ${ }^{4}$.

La legitimidad del GAFI como creador de estándares globales es un problema recurrente ya que este es un organismo intergubernamental que diseña y supervisa el régimen internacional $\mathrm{AML} / \mathrm{CFT}$, pero no es inmediatamente visible quién gobierna el GAFI y quién establece su agenda. El GAFI se ve a veces como un agente para la

\footnotetext{
${ }^{2}$ LOMBARDERO EXPÓSITO, L. M. (2008), “El grupo de acción financiera internacional: revisión del mandato (2008-2012)”, Revista de Estudios Jurídicos (Segunda Época), nº 8/2008, pp. 14-15.

${ }^{3}$ FATF (2019), Mandate approved by the Ministers and Representatives of the Financial Action Task Force, Washington DC, 12.02.2019 ("The FATF has evolved from a temporary forum to a sustained public and political commitment to fight money laundering, terrorist financing, and proliferation financing”).

${ }^{4}$ FATF (2019), Report to the G20 Leaders' Summit, June 2019.
} 
promoción de la agenda de aquellos pocos países desarrollados que habían estado involucrados en el desarrollo de las Recomendaciones originales. Sin rechazar las críticas sobre el déficit democrático y la falta de transparencia, encontramos que el argumento de "captura por agencia" (agency capture) y "subordinación hegemónica" no hace justicia al proceso de creación de normas del GAFI ${ }^{5}$. La confiabilidad del GAFI se ve reflejada en el número creciente de sus miembros, incluidos importantes actores internacionales como la Federación de Rusia (desde 2003), la República Popular de China (desde 2007), India (desde 2010), etc. Además de esto, el GAFI a menudo incorpora comentarios y posiciones de las partes interesadas, en particular los participantes del mercado financiero; por lo tanto, los Estados no son la única fuente institucional de autoridad política e innovación en el proceso del GAFI, que comprende "una interacción más amplia de procesos y prácticas de gobernanza"6 ${ }^{6}$. Estos elementos hacen que la gobernanza del GAFI sea más equilibrada y autónoma, diluyendo los vínculos con la agenda política de los Estados Unidos de América, que fueron más fuertes a principios de la década de 1990.

Otra fuente de legitimidad es la comercialización exitosa del GAFI y de sus estándares como una solución experta e imparcial avalada por un organismo especializado ${ }^{7}$. Al ser un organismo multidisciplinario que reúne a expertos en las áreas de finanzas y Derecho $^{8}$, los Estándares del GAFI incorporan la experiencia de las jurisdicciones participantes. Ampliar la base de participación en la toma de decisiones del GAFI mejoraría la legitimidad del proceso, ya que permitiría que "procesos anteriormente tecnocráticos y cerrados" se beneficien de más comentarios, información y argumentos ${ }^{9}$. Siguiendo esta lógica, el mandato del GAFI correspondiente al periodo 2012-2020 reconoció la importancia de mejorar la transparencia y la previsibilidad en la toma de decisiones y la gobernanza ${ }^{10}$. Desde entonces, el GAFI ha logrado un progreso significativo a este respecto al apoyar un diálogo constructivo con el sector privado, como en el caso del compromiso del GAFI con los sectores de la tecnología financiera y la tecnología reguladora (FinTech \& RegTech) ${ }^{11}$. La legitimación del GAFI se evidencia aún más por la aceptación de sus normas por un número creciente de jurisdicciones en los últimos 30 años, contando actualmente con 39 miembros (37

\footnotetext{
${ }^{5}$ BELAY, H. (2019), “Global Financial Regulation in the New International Economic Order”, Journal of Financial Regulation and Compliance, Vol. 27, No. 1, pp. 13-30; GHOSHRAY, S. (2015), "Compliance Convergence in FATF Rulemaking: The Conflict Between Agency Capture and Soft Law", New York Law School Law Review, Vol. 59, No. 3, pp. 521-545.

${ }^{6}$ HENG, Y., \& MCDONAGH, K. (2008), "The Other War on Terror Revealed: Global Governmentality and the Financial Action Task Force's Campaign against Terrorist Financing”, Review of International Studies, Vol. 34, No. 3, pp. 553-573.

7 HÜLSSE, R., \& KERWER, D. (2007), "Global Standards in Action: Insights from Anti-Money Laundering Regulation”, Organization, Vol. 14, No. 5, pp. 625-642.

${ }^{8}$ SPREUTELS, J. P. (1996), Le Groupe d'action financière (GAFI) et la lutte contre le blanchiment de capitaux, L'évolution de la criminalité organisée, XVIIIème Cours International de Haute Spécialisation pour les Forces de Police, Institut des hautes études de la sécurité intérieure, pp. 343-350.

${ }_{9}^{9}$ NANCE, M. (2018), "Re-thinking FATF: An Experimentalist Interpretation of the Financial Action Task Force”, Crime, Law and Social Change, Vol. 69, No. 2, pp 131-152

${ }^{10}$ FATF (2012), Ministers Renew the Mandate of the Financial Action Task Force until 2020: Declaration of the Ministers and Representatives of the Financial Action Task, Washington, DC, 20 April 2012.

${ }^{11}$ Para ver los resúmenes de las numerosas mesas redondas, reuniones y foros organizados por el GAFI e involucrando a representantes de la comunidad FinTech \& RegTech, consulte: http://www.fatfgafi.org/fintech-regtech/fatfonfintechregtech/?hf $=10 \& \mathrm{~b}=0 \& \mathrm{~s}=\mathrm{desc}$ (fatf_releasedate) [consultado el $1 \mathrm{de}$ marzo de 2020]
} 
países miembros y dos organizaciones internacionales) y varios observadores, más del doble de los 16 miembros iniciales.

\section{DIFUMINAR LA DICOTOMÍA ENTRE EL DERECHO VINCULANTE Y LOS INSTRUMENTOS DE CARÁCTER NO VINCULANTE}

El éxito del GAFI como creador de estándares y como revisor de implementación de dichos estándares se evidencia por el impacto de las 40 Recomendaciones, las notas interpretativas y las guías del GAFI sobre la legislación nacional, la legislación de la Unión Europea (UE) y el Derecho internacional tradicional. Dado el alto nivel de cumplimiento con los estándares del GAFI, se puede argumentar que han asumido un estatus cuasi-vinculante, difuminando la dicotomía entre el Derecho vinculante y los instrumentos de carácter no vinculante en el contexto del AML/CFT.

La realidad legal a nivel nacional e internacional se caracteriza progresivamente por la proliferación de potestades normativas, interventores y partes interesadas en el proceso de elaboración de normas, así como por la aparición de fuentes alternativas, como los instrumentos de carácter no vinculante ("ley blanda”, "soft law”) ${ }^{12}$. Estos instrumentos laxos (circulares, estándares, instrucciones, códigos de buen gobierno, etc.) carecen de normatividad stricto sensu, desafían la rigidez del principio de legalidad y han sido utilizados en el ámbito de la autorregulación y como una técnica legislativa para la unificación y armonización del Derecho en diversos campos. Son difíciles de clasificar en el sistema de fuentes del Derecho, porque no se fundamentan en un régimen de sanciones formales, pero crean expectativas de cumplimiento futuro y se incorporan rutinariamente en el orden legal, ya sea canalizados a normas de Derecho positivo o utilizados como herramienta de interpretación jurídica para esas normas. En consecuencia, se vuelve natural acatarlos y sus efectos, aunque indirectos y no reconocidos expresamente, son inevitables y condicionan la actividad del Estado y los actores privados.

Las 40 recomendaciones del GAFI son un documento de referencia en el proceso de creación de normas internacionales AML/CFL. Actualizados varias veces y acompañados de sus respectivas notas interpretativas, guías y mejores prácticas ${ }^{13}$, las recomendaciones cubren aspectos como la criminalización del blanqueo de capitales y de la financiación del terrorismo, la regulación del sistema financiero, el decomiso de los recursos de procedencia ilícita, la cooperación internacional, etc. Las recomendaciones constituyen instrumentos de carácter no vinculante ${ }^{14}$; por lo tanto, no

\footnotetext{
${ }^{12}$ DEL TORO HUERTA, M. I. (2006), "El fenómeno del soft law y las nuevas perspectivas del Derecho internacional”, Anuario Mexicano de Derecho Internacional, Vol. 6, pp. 513-549; SANZ R., FOLLONI A. (2017), "El soft law como fuente del derecho internacional: reflexiones desde la teoría de la complejidad”, Revista de Direito Internacional, Vol. 14/3, pp. 243-259; DI ROBILANT, A. (2006), “Genealogies of Soft Law”, American Journal of Comparative Law, Vol. 54, pp. 499-554.

13 Para el texto completo de estos documentos e información sobre las actualizaciones, ver http://www.fatf-gafi.org/publications/fatfrecommendations/documents/fatf-recommendations.html [consultado el 16 de mayo de 2020]

${ }^{14}$ GUYMON, D. C. (2000), "International Legal Mechanisms for Combating Transnational Organised Crime: The Need for a Multilateral Convention”, Berkeley Journal of International Law, Vol. 18, pp. 53101; ZAGARIS, B. / CASTILLA, S. M. (1993), "Constructing an International Financial Enforcement Subregime: The Implementation of Anti-Money-Laundering Policy”, Brooklyn Journal of International Law, Vol. 19, No. 3, pp. 871-965.
} 
tienen un efecto obligatorio y carácter jurídicamente vinculante stricto sensu según el Derecho internacional ${ }^{15}$.

Sin embargo, esta declaración no coincide con el alto nivel de cumplimiento de los miembros del $\mathrm{GAFI}^{16}$, ya que han hecho fuertes compromisos políticos para transponer las Recomendaciones a las leyes nacionales y han puesto estas en práctica con notable consistencia. Las obligaciones impuestas por un tratado internacional siguen siendo superiores a las Recomendaciones en caso de conflicto de normas y prometen una aplicación más consistente y homogénea. Sin embargo, los estándares del GAFI ofrecen las ventajas de flexibilidad y modificación más fácil y rápida. También se ha demostrado que son capaces de garantizar un cumplimiento constante, aunque todavía hay margen de mejora ${ }^{17}$. De hecho, los Estados miembros participan en el proceso de evaluación mutua y el seguimiento de la evaluación, y aceptan la evaluación del GAFI concerniente a sus marcos AML/CFT ${ }^{18}$. Luego actúan sobre esta evaluación al informar sobre los pasos que han tomado para abordar las deficiencias identificadas ${ }^{19}$.

Al mismo tiempo, las organizaciones y foros internacionales regularmente hacen referencia al trabajo del GAFI y exigen una "implementación rápida y efectiva" de sus estándares ${ }^{20}$. Por lo tanto, los Estándares del GAFI han asumido de facto un estado cuasi-vinculante en el contexto AML/CFT, desdibujando la dicotomía entre el Derecho vinculante y los instrumentos de carácter no vinculante ${ }^{21}$. Como dijo Deng Xiaoping, ex líder de la República Popular de China: "No importa que el gato sea blanco o sea negro. Mientras cace ratones, es un buen gato", lo que en el caso del GAFI se traduce en un aumento de la eficiencia del régimen AML/CFT en todo el mundo.

El paradigma del GAFI es un ejemplo de gobernanza 'nueva' o 'experimental' ${ }^{22}$ que promete más flexibilidad, es decir, una adaptación más rápida a nuevas realidades y una transposición más rápida de reglas y contramedidas exitosas. Los instrumentos de carácter no vinculante son más adecuados para acomodar la diversidad nacional que los tratados internacionales, promoviendo la convergencia de normas de una manera más flexible ${ }^{23}$. Por esta razón, el GAFI alienta a sus miembros a "hacer uso de la flexibilidad de las Recomendaciones del GAFI"24. Los planes operativos del GAFI, como el plan

15 TURNER, N. (2015), “The Financial Action Task Force: International Regulatory Convergence Through Soft Law”, New York Law School Law Review, Vol. 59, pp. 548-559.

${ }^{16}$ CASSANI, U. (2008), "L’internationalisation du droit pénal économique et la politique criminelle de la Suisse : la lutte contre le blanchiment d'argent”, Revue de droit suisse, Vol. 127, No. II/2, pp. 227-398

17 MEKPOR, E. (2019), "Anti-Money Laundering and Combating the Financing of Terrorism Compliance”, Journal of Money Laundering Control, Vol. 22, No. 3, pp. 451-471; MEKPOR, E., ABOAGYE, A. WELBECK, J. (2018), "The Determinants of Anti-Money Laundering Compliance among the Financial Action Task Force (FATF) Member States”, Journal of Financial Regulation and Compliance, Vol. 26, No. 3, pp. 442-459.

${ }^{18}$ P.ej. FATF, Spain's progress to improve effectiveness in combating money laundering and terrorist financing, Paris, 04.12.2019; EUROPA PRESS (2019), El GAFI sitúa a España entre los mejores países contra el blanqueo de capitales y financiación al terrorismo, Madrid, 04.12.2019.

19 P. ej. FATF (2018), Anti-money laundering and counter-terrorist financing measures - Spain, 1st Regular Follow-up Report \& Technical Compliance Re-Rating, Paris, 20.03.2018.

${ }^{20}$ Ver p. ej.: Hamburg G20 Summit (July 2017), G20 Leaders’ Statement on Countering Terrorism.

21 LICHTENSTEIN, C. (2001), “Hard Law v. Soft Law: Unnecessary Dichotomy?”, The International Lawyer, Vol. 35, No. 4, pp. 1433-1441.

${ }^{22}$ NANCE, M., COTTRELL, M. (2014), “A Turn Toward Experimentalism? Rethinking Security and Governance in the Twenty-First Century”, Review of International Studies, Vol. 40, No. 2, pp. $277-301$.

23 ABBOTT, K. W., \& SNIDAL, D. (2000), "Hard and Soft Law in International Governance", International Organization, Vol. 54, No. 3, pp. 421-456.

${ }^{24}$ FATF (2017), Revised Guidance on AML/CFT and Financial Inclusion, Paris. 
operativo contra la financiación del terrorismo del 2018, están diseñados para permanecer flexibles y adaptables de manera que puedan abordar vulnerabilidades a medida que se observan y enfrentar cualquier amenaza emergente ${ }^{25}$. Otro ejemplo de la flexibilidad del GAFI es la adopción del llamado "enfoque basado en el riesgo" ("riskbased approach"). En este subproceso, cada jurisdicción debe identificar y comprender los riesgos de AML/CFT a los que está expuesta, a fin de adaptar las reglas AML/CFT a sus características jurisdiccionales específicas y aplicar medidas de una manera que asegure la mitigación de estos riesgos. Por lo tanto, la "ley blanda" se suaviza aún más y se convierte en un agente casi invisible que mantiene la ley nacional "bajo control", aumentando aún más la convergencia de las normas.

A este respecto, el GAFI también podría verse como una "organización global de creación de conocimiento común" ${ }^{26}$. Siguiendo un modelo de creación e intercambio de conocimiento, el GAFI transmite información relacionada con las AML/CFT a organizaciones internacionales, miembros del GAFI y, en última instancia, a Unidades de Inteligencia Financiera, agencias de aplicación de la ley y agencias de supervisión en cada Estado miembro.

\section{GARANTIZAR LA IMPLEMENTACIÓN UNIVERSAL DE LOS ESTÁNDARES DEL GAFI}

Para garantizar la transposición de sus Normas a nivel nacional, el GAFI examina las leyes y prácticas de los países miembros de manera regular. El sistema de evaluación mutua ("mutual evaluation”) ${ }^{27}$ desarrollado para este fin se ha convertido en un proceso de referencia para monitorear la implementación de instrumentos internacionales. El sistema de monitoreo se basa en revisiones por pares realizadas de manera continua para cada miembro. Los expertos del GAFI revisan la implementación de los estándares y proporcionan un análisis exhaustivo de las leyes y prácticas nacionales en el área AML/CFT; en última instancia, evalúan la efectividad del sistema nacional AML/CFT (cumplimiento técnico y evaluación de efectividad).

Además del GAFI, organismos regionales al estilo del mismo respaldan los estándares del GAFI y evalúan el cumplimiento por parte de sus miembros mediante evaluaciones mutuas de acuerdo con una metodología comúnmente acordada ${ }^{28}$. El GAFI y los organismos regionales tipo GAFI ("FATF-style organisations") son organizaciones independientes sin jerarquía entre ellas, aunque los Principios de alto nivel de 2012 aclaran que el GAFI es el único organismo normativo y el tutor y árbitro de la aplicación de sus estándares ${ }^{29}$. Juntos, el GAFI y la red de organismos regionales cubren 205 países, lo que hace que los estándares adoptados por el GAFI sean verdaderamente globales en el contexto AML/CFT.

\footnotetext{
25 FATF (2018), Annual Report 2017-2018, en $\underline{\text { www.fatf- }}$ gafi.org/publications/fatfgeneral/documents/annual-report-2017-2018.html [consultado el 16 de mayo de 2020]

26 YASAKA, N. (2017), "Knowledge Management in International Cooperation for Anti-Money Laundering”, Journal of Money Laundering Control, Vol. 20, No. 1, pp. 27-34.

${ }^{27}$ FATF (2013), Methodology for assessing technical compliance with the FATF Recommendations and the Effectiveness of AML/CFT Systems [as updated in February 2019].

28 FATF (2019), Consolidated processes and procedures for mutual evaluations and follow up ("Universal Procedures").

29 FATF (2012), High-Level Principles for the Relationship between the FATF and the FATF-Style Regional Bodies, updated February 2019, Paris.
} 
Además del proceso de evaluaciones mutuas, el GAFI ha recurrido a la práctica de incluir en la lista negra ("blacklisting") a las jurisdicciones que no pueden combatir el blanqueo de capitales y la financiación del terrorismo ${ }^{30}$. Hacer una lista pública de estas jurisdicciones ha sido un medio para presionar y obligarlas a hacer las reformas necesarias y abordar sus debilidades. De hecho, de las 68 jurisdicciones con sistemas AML/CFT débiles que el GAFI ha identificado, 55 han adoptado reformas, lo que resultó en su eventual eliminación de la lista negra. Curiosamente, en algunas ocasiones los Estados no pertenecientes al GAFI han adoptado sus reglas y las han cumplido incluso antes de que el GAFI recurriera a las listas negras, lo que demuestra aún más la eficacia de este método.

Asimismo, el GAFI se ha esforzado por mejorar la legitimación de sus procesos para garantizar mejor el cumplimiento poniendo énfasis en la experiencia y la participación ${ }^{31}$. En el marco de la cuarta ronda de evaluaciones mutuas en curso, las calificaciones de evaluación consolidadas muestran que el cumplimiento para la mayoría de las jurisdicciones es muy satisfactorio, con avances en varios países con respeto a la tercera ronda de evaluaciones mutuas ${ }^{32}$. Además, algunos casos de deficiencias de cumplimiento pueden explicarse por el hecho de que los estándares AML/CFT que se evalúan en la cuarta ronda se han actualizado y se han vuelto más estrictos en comparación con los examinados en la tercera. El principal desafío que queda para las futuras rondas de evaluación es cambiar el enfoque del GAFI con respeto a la efectividad de cumplimiento técnico ("marcar la casilla") favoreciendo un modelo orientado a resultados ${ }^{33}$.

\section{EXPANSIÓN DE LOS ESTÁNDARES DEL GAFI}

En los últimos 30 años, el alcance y la cobertura de la "ley blanda" del GAFI se ha expandido considerablemente. Las 40 Recomendaciones, formuladas en 1990, fueron revisadas por primera vez en 1996 para adaptarse al fenómeno cambiante del blanqueo de capitales. Entre las mejoras introducidos en 1996 está la extensión del alcance del blanqueo de capitales para incluir delitos graves, mucho más allá de los delitos relacionados con el tráfico estupefacientes. Una revisión más exhaustiva tuvo lugar en 2003, que combinaba las 40 Recomendaciones revisadas con 9 Recomendaciones especiales sobre el financiamiento del terrorismo; tales modificaciones tenían como objetivo fortalecer las medidas AML/CFT. El desarrollo de estándares contra el financiamiento de la proliferación de armas de destrucción masiva también se ha convertido en parte de la agenda del GAFI desde el mandato

\footnotetext{
${ }^{30}$ MORSE, J. (2019), "Blacklists, Market Enforcement, and the Global Regime to Combat Terrorist Financing”, International Organization, Vol. 73, No. 3, pp. 511-545; STESSENS, G. (2001), "The FATF 'Black List' of Non-Cooperative Countries or Territories”, Leiden Journal of International Law, Vol. 14, No. 1, pp. 199-207; AGUIRRE QUEZADA, J. B. (2017), "Panorama del lavado de dinero a escala internacional”, Revista Internacional de Ciencias del Estado y de Gobierno, vol. 1, no 1-2, pp.168-186, p. 171.

${ }^{31}$ KERWER, D., HÜLSSE, R. (2011), "How International Organizations Rule the World: The Case of the Financial Action Task Force on Money Laundering”, Journal of International Organizations Studies, Vol. 2, No. 1, pp. 50-67

32 FATF (2019), Consolidated Table of Assessment Ratings, Updated 30 April 2020, consulte: https://www.fatf-gafi.org/media/fatf/documents/4th-Round-Ratings.pdf [consultado el 4 de junio de 2020] ${ }_{33}$ POL, R. (2018), “Anti-Money Laundering Effectiveness: Assessing Outcomes or Ticking Boxes?”, Journal of Money Laundering Control, Vol. 21, No. 2, pp. 215-230.
} 
correspondiente al periodo de 2012-2020. Estas normas se relacionan con la implementación de las resoluciones del Consejo de Seguridad de las Naciones Unidas (CSNU) sobre la no proliferación de armas de destrucción masiva, como la resolución 1540 (2004) y las resoluciones dirigidas a países específicos, como la resolución 1803 (2008) sobre Irán y la resolución 1874 (2009) sobre Corea del Norte. En varios aspectos, el GAFI ha actuado en el territorio del Derecho internacional público al duplicar parcialmente el trabajo de la ONU, como en el caso de las resoluciones antes mencionadas, las resoluciones del CSNU contra la financiación del terrorismo y el Convenio Internacional para la Represión de la Financiación del Terrorismo.

Los límites de esta expansión han sido enarbolados por distintos entes debido a la evolución de las amenazas y vulnerabilidades asociadas con el blanqueo de capitales, la financiación del terrorismo y financiación de la proliferación de armas de destrucción masiva, que requieren respuestas rápidas y el desarrollo de nuevos estándares y principios. P. ej. el GAFI ha reconocido los nuevos desafíos regulatorios y de supervisión en el campo de FinTech y RegTech y ha organizado un diálogo con el sector privado como parte del Foro consultivo del sector privado del GAFI - siendo los Principios rectores de San José 2017 el primer producto de este diálogo ${ }^{34}$. El trabajo reciente del GAFI sobre la regulación y supervisión de los activos virtuales y los proveedores de servicios de activos virtuales, en particular la enmienda de la Recomendación 15 del GAFI y la adopción de una Nota interpretativa, constituyen otro ejemplo de la expansión de los estándares del GAFI, que deben seguir el ritmo de rápidos desarrollos en el campo de las finanzas y la tecnología. El establecimiento de estándares del GAFI es un trabajo en progreso que busca estudiar y abordar la evolución de los métodos utilizados por los delincuentes en todo el mundo. Por lo tanto, la expansión de los estándares del GAFI es inevitable, ya que constantemente surgen nuevas amenazas y desafíos en el contexto AML/CFT.

\section{SINERGIAS ENTRE EL DERECHO NACIONAL, EL DERECHO INTERNACIONAL Y LAS NORMAS DEL GAFI}

La primera versión de las Recomendaciones del GAFI ha sido fuertemente influenciada por las legislaciones nacionales contra el blanqueo, como la legislación de los Estados Unidos. Sin embargo, no le está haciendo justicia al GAFI ver su evolución conformada únicamente por una agenda hegemónica. En cambio, los estándares del GAFI deben ser vistos como un ejercicio continuo de Derecho comparado, por el cual las normas nacionales exitosas pueden convertirse en estándares globales a través de su aceptación por los miembros del GAFI y su incorporación a los estándares. Las jurisdicciones miembros del GAFI se han comprometido a participar activamente en forma de reuniones, informes, iniciativas con el sector privado, mejores prácticas, etc., contribuyendo así a la configuración de los estándares ${ }^{35}$.

Además, la evolución del GAFI también ha sido moldeada por el Derecho internacional, ya que sus textos a menudo se refieren a instrumentos de Derecho internacional (como la Convención de las Naciones Unidas contra el tráfico ilícito de estupefacientes y sustancias psicotrópicas de 1988, la Convención de las Naciones

\footnotetext{
34 San Jose Guiding Principles (2017), en http://www.fatfgafi.org/publications/fatfgeneral/documents/fatf-fintech-regtech-forum-may-2017.html [consultado el 4 de junio de 2020]

${ }^{35}$ FATF (2019), Report to the G20 Leaders’ Summit, June 2019
} 
Unidas contra la Delincuencia Organizada Transnacional de 2000, la Convención de las Naciones Unidas contra la Corrupción de 2003 y el Convenio internacional de Naciones Unidas para la represión de la financiación del terrorismo de 1999) y recomiendan su ratificación sin reservas, como en el caso de la Recomendación 36 del GAFI.

Otro subproceso importante es el impacto de los estándares del GAFI en la ley dura, es decir, el endurecimiento del marco regulatorio "blando". Las normas del GAFI se convierten en leyes estrictas cuando se integran en la legislación nacional, el Derecho de la Unión Europea o el Derecho internacional público ${ }^{36}$. Las 40 Recomendaciones se han convertido en el texto de referencia para las estrategias contra el blanqueo de capitales y la financiación del terrorismo en todo el mundo y han influido en el trabajo de organismos internacionales, como el Consejo de Europa, especialmente el Convenio núm. 198, como lo demuestra el Artículo 13. Las Recomendaciones del GAFI también influyen en el trabajo de la Unión Europea ${ }^{37}$, desde la redacción de la primera Directiva contra el blanqueo de capitales (Directiva 91/308) hasta la quinta Directiva contra el blanqueo de capitales (Directiva 2018/843) y muchos otros instrumentos, como la Directiva sobre el decomiso del producto del delito (Directiva 2014/42), el Reglamento sobre el reconocimiento mutuo de las resoluciones de decomiso (Reglamento 2018/1805), etc. ${ }^{38}$ A nivel de la ONU, hay varios ejemplos de los instrumentos explícitamente enfatizando el papel esencial del GAFI en el establecimiento de estándares globales para prevenir y combatir el blanqueo de capitales, la financiación del terrorismo, la financiación de la proliferación de armas de destrucción masiva y alentando a los Estados miembros de la ONU a cooperar activamente con el GAFI ${ }^{39}$. Por lo tanto, los estándares del GAFI pueden incorporarse en última instancia a la ley dura y resurgir como obligaciones vinculantes en el Derecho nacional, de la UE e internacional. Es importante resaltar que estas sinergias son constructivas y positivas, siempre que el GAFI, las organizaciones internacionales y las jurisdicciones nacionales busquen la coherencia de las normas y eviten la duplicación innecesaria y derrochadora del trabajo.

Dado que el objetivo de la cohesión no es un fin en sí mismo, los estándares y el modelo analítico utilizado por el GAFI deberían permitir aportes, críticas y mejoras. Por ejemplo, se ha argumentado que este modelo analítico se basa en una clasificación temporal del banqueo en tres fases (colocación, enmascaramiento e integración), pero en realidad los actos a menudo carecen de un carácter secuencial y no necesariamente terminan en blanqueo ${ }^{40}$. En el mismo sentido, se ha argumentado que la etapa final debe

\footnotetext{
${ }^{36}$ FLORES, A. R., GONZÁLEZ, F. (2017), "La lucha contra el blanqueo de capitales y la financiación del terrorismo tras la adhesión de España a la UE”, Revista Universitaria Europea, n 26, pp. 91-120.

37 SPREUTELS, J. P. / SCOHIER, C. (1998), "La prévention du blanchiment de capitaux: évolutions récentes”, Revue de Droit de l’ULB, Vol. 1997-1, pp. 165 ss; GILMORE, W. (2005), L'argent sale: L'évolution des mesures internationales de lutte contre le blanchiment de capitaux et le financement du terrorisme, Editions du Conseil de l'Europe.

38 PAVLIDIS, G. (2019), "Learning from Failure: Cross-Border Confiscation in the EU”, Journal of Financial Crime, Vol. 26, No. 3, pp. 683-691; HOUSE OF LORDS (2009), Money Laundering and the Financing of Terrorism, European Union Committee, 19th Report of Session 2008-09, HL Paper 132-I, 22.07.2009; MITSILEGAS, V., \& GILMORE, B. (2007), “The EU Legislative Framework Against Money Laundering and Terrorist Finance: A Critical Analysis in the Light of Evolving Global Standards”, International and Comparative Law Quarterly, vol. 56, No. 1, pp. 119-140.

${ }^{39}$ Resolución 2462 (2019) del Consejo de Seguridad, 28 de marzo de 2019.

40 PRIETO DEL PINO, A. M., GARCÍA MAGNA, D. I., MARTÍN PARDO, A. (2010), "La deconstrucción del concepto de blanqueo de capitales”, InDret, nº 3/2010, p. 5.
} 
subdividirse aún más, para evitar corresponder a la realidad del banqueo ${ }^{41}$. Tales críticas, desafiando el modelo analítico del GAFI y la idea clásica del banqueo como un proceso trifásico, deben ser consideradas por el GAFI, cuya responsabilidad como creador de estándares globales no es imponer modelos dogmáticos y poco realistas en sus Estados miembros, sino construir su paradigma sobre aportes y propuestas constructivas.

\section{VI. ¿CREACIÓN DE NORMAS DEL GAFI O UNA NUEVA CONVENCIÓN INTERNACIONAL?}

El Derecho internacional y los instrumentos de "ley blanda" para combatir el blanqueo de capitales, la corrupción y la delincuencia organizada transnacional se superponen en cierta medida. De hecho, las disposiciones sobre el blanqueo de capitales y el decomiso del producto del delito se pueden encontrar en la Convención de las Naciones Unidas contra el tráfico ilícito de estupefacientes y sustancias psicotrópicas de 1988, la Convención de las Naciones Unidas contra la delincuencia organizada transnacional de 2000, la Convención de las Naciones Unidas contra la corrupción de 2003, las convenciones regionales como los Convenios del Consejo de Europa $\mathrm{N}^{\mathrm{o}} 141 \mathrm{y}$ $\mathrm{N}^{\mathrm{o}}$ 198, las cinco Directivas de la UE contra el blanqueo de dinero, etc. Cada iniciativa es producto de un largo proceso de negociación y compromiso, y es parte de una evolución legal que refleja el impulso político de la época. Por lo tanto, la fragmentación y la falta de estandarización son hasta cierto punto inevitables, ya que las Naciones Unidas no han estado dispuestas a "aprovechar más su función de coordinadora a nivel mundial de los distintos grupos interesados" 42 .

¿Constituiría un instrumento global sobre el blanqueo de capitales y el decomiso una solución más coherente que una serie de instrumentos fragmentados que abordan aspectos específicos del mismo problema? La idea de crear una convención global sobre estos temas parece demasiado ambiciosa, ya que es probable que su adopción enfrente dificultades y demoras considerables, como suele ser el caso con los instrumentos internacionales existentes ${ }^{43}$. Por esta razón, el enfoque del GAFI, basado en la "ley blanda", parece tener mejores posibilidades de éxito. Esta declaración se ve reforzada por el hecho de que la visibilidad del GAFI en los organismos de gobernanza global ha aumentado gradualmente a través de la participación del GAFI en varios foros internacionales, incluso en el G7 / G20, las Naciones Unidas y sus organismos y los organismos regionales al estilo del GAFI. Finalmente, el nivel de cumplimiento de los estándares del GAFI a nivel mundial es muy satisfactorio, como lo demuestran las calificaciones de evaluación consolidadas ${ }^{44}$. Por lo tanto, el enfoque del GAFI no está en desventaja en comparación con las obligaciones del Derecho internacional, cuyo cumplimiento no siempre es un hecho dado y "la capacidad del sistema para

\footnotetext{
${ }^{41}$ VAN KONINGSVELD, J. (2013), “You Don’t See It, Until You Understand It: Rethinking the Stages of the Money Laundering Process to Make Enforcement More Effective”, Research Handbook on Money Laundering, Edward Elgar Publishing, pp. 435-451

42 DE FARAMIÑÁN GILBERT, J. M. (2005), "Las necesarias modificaciones de las Naciones Unidas en un mundo globalizado (en su sesenta aniversario)”, Revista electrónica de estudios internacionales, $\mathrm{n}^{\circ}$ 10, p. 5.

43 PAVLIDIS, G. (2012), Confiscation internationale: instruments internationaux, droit de l'Union européenne, droit suisse, Schulthess, Genève, p. 101.

${ }^{44}$ FATF (2019), Consolidated Table of Assessment Ratings, Updated 30 April 2020, consulte:

https://www.fatf-gafi.org/media/fatf/documents/4th-Round-Ratings.pdf [consultado el 4 de junio de 2020]
} 
autocorregirse y autoejecutarse es limitada”, creando brechas entre aspiraciones y realidad $^{45}$.

\section{CONCLUSIONES}

Se puede evidenciar una proliferación de iniciativas internacionales en el contexto AML/CFT después la adopción de la Convención contra el tráfico ilícito de estupefacientes en 1988. Claramente, tal movilización de la comunidad internacional para la introducción de controles AML/CFT fue necesaria para evitar la explotación de la globalización financiera por parte de delincuentes y organizaciones criminales ${ }^{46}$. La proliferación de iniciativas ha sido acompañada por una multiplicación de actores en el proceso de establecimiento de normas ${ }^{47}$, una tendencia que es cada vez más característica del Derecho penal económico y relativiza, en cierta medida, el poder normativo del Estado. Por lo tanto, el legislador nacional debe tener en cuenta el trabajo realizado y las propuestas presentadas por organizaciones internacionales, legisladores de otros Estados, ONG y la sociedad civil, partes interesadas del sector financiero, etc. En este proceso de establecimiento de normas, los instrumentos de "leyes blandas" están influyendo cada vez más en las políticas nacionales de lucha contra el crimen; como ejemplo, destaca el trabajo del GAFI en la lucha contra el blanqueo de capitales, la financiación del terrorismo y la financiación y proliferación de armas de destrucción masiva.

Aunque los estándares del GAFI no tienen ninguna obligatoriedad explícita y formal bajo el Derecho internacional, sus medios de acción, especialmente el proceso de evaluación mutua y el proceso de inclusión en listas negras, han demostrado ser persuasivos y efectivos para garantizar el cumplimiento en el contexto AML/CFT. Sin embargo, la efectividad de las herramientas del GAFI no es solo el resultado de políticas coercitivas ("naming and shaming” / "nombrar y avergonzar"), sino que también se puede atribuir a los mayores esfuerzos del GAFI para mejorar su legitimidad y transparencia y aumentar la participación de las partes interesadas. Estos objetivos deben ser perseguidos aún más enérgicamente por el GAFI, a fin de garantizar la entrega de estándares de calidad que gocen de aceptación e implementación internacional.

\section{BIBLIOGRAFÍA}

ABBOTT, K. W., \& SNIDAL, D. (2000), "Hard and Soft Law in International Governance”, International Organization, vol. 54, nº 3, pp. 421-456.

AGUIRRE QUEZADA, J. B. (2017), "Panorama del lavado de dinero a escala internacional”, Revista Internacional de Ciencias del Estado y de Gobierno, vol. 1, no 1-2, pp.168-186, p. 171.

\footnotetext{
${ }^{45}$ COGAN, J. (2006), “Noncompliance and the International Rule of Law”, Yale Journal of International Law, Vol. 31, No. 1, pp. 189-210.

${ }^{46}$ GÁLVEZ BRAVO, R. (2017), Los modus operandi en las operaciones de blanqueo de capitales, Wolters Kluwer, Barcelona, p. 237; DEL CID GÓMEZ, J. M. (2007), Blanqueo internacional de capitales: Cómo detectarlo y prevenirlo, Ediciones Deusto, Barcelona, 2007, pp. 19-30.

${ }^{47}$ CASSANI, U. (2008), "L’internationalisation du droit pénal économique et la politique criminelle de la Suisse : la lutte contre le blanchiment d'argent”, Revue de droit suisse, Vol. 127, No. II/2, pp. 227-398.
} 
BELAY, H. (2019), "Global Financial Regulation in the New International Economic Order”, Journal of Financial Regulation and Compliance, vol. 27, $\mathrm{n}^{\circ}$. 1, pp. 1330

CASSANI, U. (2008), “L’internationalisation du droit pénal économique et la politique criminelle de la Suisse: la lutte contre le blanchiment d'argent”, Revue de droit suisse, vol. $127, \mathrm{n}^{\circ} . \mathrm{II} / 2$, pp. 227-398

CASSANI, U. (2008), "L’internationalisation du droit pénal économique et la politique criminelle de la Suisse la lutte contre le blanchiment d'argent", Revue de droit suisse, vol. 127, $\mathrm{n}^{\circ}$. II/2, pp. 227-398.

COGAN, J. (2006), “Noncompliance and the International Rule of Law”, Yale Journal of International Law, vol. 31, nº. 1, pp. 189-210.

DE FARAMIÑÁN GILBERT, J. M. (2005), "Las necesarias modificaciones de las Naciones Unidas en un mundo globalizado (en su sesenta aniversario)”, Revista electrónica de estudios internacionales, No. 10.

DEL CID GÓMEZ, J. M. (2007), Blanqueo internacional de capitales: Cómo detectarlo y prevenirlo, Ediciones Deusto, Barcelona, 2007.

DEL TORO HUERTA, M. I. (2006), "El fenómeno del soft law y las nuevas perspectivas del Derecho internacional”, Anuario Mexicano de Derecho Internacional, vol. 6, pp. 513-549.

DI ROBILANT, A. (2006), "Genealogies of Soft Law”, American Journal of Comparative Law, vol. 54, pp. 499-554.

FATF (2012), High-Level Principles for the Relationship between the FATF and the FATF-Style Regional Bodies, updated February 2019, Paris.

FATF (2012), Ministers Renew the Mandate of the Financial Action Task Force until 2020: Declaration of the Ministers and Representatives of the Financial Action Task, Washington, DC, 20 April 2012.

FATF (2013), Methodology for assessing technical compliance with the FATF Recommendations and the Effectiveness of AML/CFT Systems [as updated in February 2019].

FATF (2017), Revised Guidance on AML/CFT and Financial Inclusion.

FATF (2019), Consolidated processes and procedures for mutual evaluations and follow up ("Universal Procedures").

FATF (2019), Mandate approved by the Ministers and Representatives of the Financial Action Task Force, Washington DC, 12.02.2019

FATF (2019), Report to the G20 Leaders' Summit, June 2019

FLORES, A. R., GONZÁLEZ, F. (2017), "La lucha contra el blanqueo de capitales y la financiación del terrorismo tras la adhesión de España a la UE”, Revista Universitaria Europea, n 26, pp. 91-120.

GÁLVEZ BRAVO, R. (2017), Los modus operandi en las operaciones de blanqueo de capitales, Wolters Kluwer, Barcelona

GHOSHRAY, S. (2015), "Compliance Convergence in FATF Rulemaking: The Conflict Between Agency Capture and Soft Law”, New York Law School Law Review, vol. 59, nº. 3, pp. 521-545.

GILMORE, W. (2005), L'argent sale: L'évolution des mesures internationales de lutte contre le blanchiment de capitaux et le financement du terrorisme, Editions du Conseil de l'Europe. 
GUYMON, D. C. (2000), "International Legal Mechanisms for Combating Transnational Organised Crime: The Need for a Multilateral Convention”, Berkeley Journal of International Law, vol. 18, pp. 53-101

HENG, Y., \& MCDONAGH, K. (2008), “The Other War on Terror Revealed: Global Governmentality and the Financial Action Task Force's Campaign against Terrorist Financing”, Review of International Studies, vol. 34, nº. 3, pp. 553-573.

HOUSE OF LORDS (2009), Money Laundering and the Financing of Terrorism, European Union Committee, 19th Report of Session 2008-09, HL Paper 132-I, 22.07.2009

HÜLSSE, R., \& KERWER, D. (2007), “Global Standards in Action: Insights from Anti-Money Laundering Regulation”, Organization, vol. 14, nº. 5, pp. 625-642.

KERWER, D., HÜLSSE, R. (2011), "How International Organizations Rule the World: The Case of the Financial Action Task Force on Money Laundering”, Journal of International Organizations Studies, vol. 2, $\mathrm{n}^{\circ}$. 1, pp. 50-67

LICHTENSTEIN, C. (2001), “Hard Law v. Soft Law: Unnecessary Dichotomy?”, The International Lawyer, Vol. 35, No. 4, pp. 1433-1441.

LOMBARDERO EXPÓSITO, L. M. (2008), "El grupo de acción financiera internacional: revisión del mandato (2008-2012)”, Revista de Estudios Jurídicos (Segunda Época), no 8/2008.

MEKPOR, E. (2019), "Anti-Money Laundering and Combating the Financing of Terrorism Compliance”, Journal of Money Laundering Control, vol. 22, $\mathrm{n}^{\circ} .3$, pp. 451-471

MEKPOR, E., ABOAGYE, A. WELBECK, J. (2018), “The Determinants of AntiMoney Laundering Compliance among the Financial Action Task Force (FATF) Member States”, Journal of Financial Regulation and Compliance, vol. 26, $\mathrm{n}^{\circ}$. 3, pp. 442-459.

MITSILEGAS, V., \& GILMORE, B. (2007), “The EU Legislative Framework Against Money Laundering and Terrorist Finance: A Critical Analysis in the Light of Evolving Global Standards”, International and Comparative Law Quarterly, vol. 56, no. 1, pp. 119-140.

MORSE, J. (2019), "Blacklists, Market Enforcement, and the Global Regime to Combat Terrorist Financing”, International Organization, Vol. 73, No. 3, pp. 511-545

NANCE, M. (2018), "Re-thinking FATF: An Experimentalist Interpretation of the Financial Action Task Force”, Crime, Law and Social Change, vol. 69, nº. 2, pp 131-152

NANCE, M., COTTRELL, M. (2014), “A Turn Toward Experimentalism? Rethinking Security and Governance in the Twenty-First Century”, Review of International Studies, vol. 40, nº. 2, pp. 277-301.

PAVLIDIS, G. (2012), Confiscation internationale: instruments internationaux, droit de l'Union européenne, droit suisse, Schulthess, Genève.

PAVLIDIS, G. (2019), "Learning from Failure: Cross-Border Confiscation in the EU”, Journal of Financial Crime, Vol. 26, No. 3, pp. 683-691

POL, R. (2018), "Anti-Money Laundering Effectiveness: Assessing Outcomes or Ticking Boxes?”, Journal of Money Laundering Control, vol. 21, nº. 2, pp. 215230.

PRIETO DEL PINO, A. M., GARCÍA MAGNA, D. I., MARTÍN PARDO, A. (2010), “La deconstrucción del concepto de blanqueo de capitales”, InDret, no 3/2010, pp. 1-36. 
SANZ R., FOLLONI A. (2017), "El soft law como fuente del derecho internacional: reflexiones desde la teoría de la complejidad”, Revista de Direito Internacional, vol. 14/3, pp. 243-259.

SPREUTELS, J. P. (1996), Le Groupe d'action financière (GAFI) et la lutte contre le blanchiment de capitaux, L'évolution de la criminalité organisée, XVIIIème Cours International de Haute Spécialisation pour les Forces de Police, Institut des hautes études de la sécurité intérieure.

SPREUTELS, J. P. / SCOHIER, C. (1998), “La prévention du blanchiment de capitaux: évolutions récentes”, Revue de Droit de l’ULB, Vol. 1997-1, pp. 165 ss

STESSENS, G. (2001), “The FATF 'Black List' of Non-Cooperative Countries or Territories”, Leiden Journal of International Law, vol. 14, nº. 1, pp. 199-207

TURNER, N. (2015), “The Financial Action Task Force: International Regulatory Convergence Through Soft Law”, New York Law School Law Review, vol. 59, pp. 548-559.

VAN KONINGSVELD, J. (2013), “You Don’t See It, Until You Understand It: Rethinking The Stages of the Money Laundering Process to Make Enforcement More Effective”, Research Handbook on Money Laundering, Edward Elgar Publishing, pp. 435-451.

YASAKA, N. (2017), "Knowledge Management in International Cooperation for AntiMoney Laundering”, Journal of Money Laundering Control, vol. 20, nº 1 , pp. 27-34.

ZAGARIS, B. / CASTILLA, S. M. (1993), "Constructing an International Financial Enforcement Subregime: The Implementation of Anti-Money-Laundering Policy”, Brooklyn Journal of International Law, vol. 19, nº. 3, pp. 871-965. 